\title{
Targeting Chaos System via Minimum Principle Control
}

\author{
Yunzhong Song \\ College of Electrical Engineering and Automation, Henan Polytechnic University, 2001 Century Avenue, Jiaozuo \\ 454003, P.R.China \\ Ziyi Fu \\ College of Electrical Engineering and Automation, Henan Polytechnic University, 2001 Century Avenue, Jiaozuo \\ 454003, P.R.China \\ Fuzhong Wang \\ College of Electrical Engineering and Automation, Henan Polytechnic University, 2001 Century Avenue, Jiaozuo \\ 454003, P.R.China \\ E-mail: songhpu@126.com,fuzy@hpu.edu.cn,wangfzh@hpu.edu.cn \\ www.hpu.edu.cn
}

\begin{abstract}
Chaos targeting via Minimum Principle Control (MPC) was suggested here, to be unique, the targeting via MPC can not only stabilize all the non-stable equilibrium when the control was introduced in the first or the second equation, but also does the extra added control in the third equation, with the cost of switching control direction.
\end{abstract}

Keywords: chaos system, Minimum Principle Control (MPC), Hamiltonian function, switching control

\section{Introduction}

An scalar and inequality constrained control method has been introduced to the well known chaotic Lorenz system by $\mathrm{Yu}-\mathrm{Chu}$ Tian et al which appeared in Physics Letters A296(2002)87-90, please referring to Reference 1. The resulting control law is bang-bang one and can stabilize the unstable equilibrium, two cases such that control action added at the first and second equations of the Lorenz system are reported. And Professor Song, together with the other authors, has addressed the tough obstacle that when the control action was added in the third equation, whereas the Tian's method was out of control, please referring to Reference 2. Since then, seldom results of constrained control to chaos system were reported and extended. In this paper, we will extend the suggested strategy to another chaos system, i.e. the Chen chaos system, please referring to Reference 3. Besides the control action being added in the first or the second equation, we will consider the case, where control action added on the third equation. Thanks to the uniqueness of the nonlinear system, different systems may express different behavior, so do the Chen chaos system; and even further, the switching surface resulted from the Hamiltonian function and the Minimum Principle Control (MPC) will also be distinct from each other, without saying the parameters selection of the controller. And the most striking point is with the completion of Chen chaos system control via MPC strategy, the belief in Lorenz family chaos system 
control will be enhanced, and the wait to go style of chaos system control is re-aroused to be the first candidate when the other strategies fail.

The rest of the paper is as follows. First, some preliminaries about Chen chaos system and MPC with constraints will be glimpsed; and then comes the main results in the third part; the conclusion and some prospects will be provided in the end.

\section{Preliminaries}

\subsection{Glimpse of Chen Chaos System}

For Chen chaos system

$$
\begin{aligned}
& \dot{x}_{1}=f_{1}\left(x_{1}, x_{2}, x_{3}\right)=a\left(x_{2}-x_{1}\right), \\
& \dot{x}_{2}=f_{2}\left(x_{1}, x_{2}, x_{3}\right)=(c-a) x_{1}-x_{1} x_{3}+c x_{2}, \\
& \dot{x}_{3}=f_{3}\left(x_{1}, x_{2}, x_{3}\right)=x_{1} x_{2}-b x_{3} .
\end{aligned}
$$

With parameters as $a=35, b=3, c=28$, the Chen chaos system will demonstrate chaotic behavior and it has three different unstable equilibriums.

$$
\begin{aligned}
x_{e}^{(1)}= & {[0,0,0]^{T}, } \\
x_{e}^{(2,3)}= & {[ \pm \sqrt{(2 c-a) b}, \pm \sqrt{(2 c-a) b}, 2 c-a] } \\
= & {[ \pm 7.9373, \pm 7.9373,21] . }
\end{aligned}
$$

\subsection{Glimpse of Constrained Control via MPC}

Consider continuous and nonlinear chaotic system

$$
\dot{x}=f(x), x\left(t_{0}\right)=x_{0}, 0=\dot{x}_{e}=f\left(x_{e}\right),
$$

where $x \in R^{n}, f \in R^{n}, t_{0}$ is the initial time constant. And $x_{e}$ demonstrates the equilibrium of the system, the introduction of control action aims to drive the unstable equilibrium into stable states. Assumed we introduce one constrained scalar control action to system (3), the system under control will turn into

$$
\dot{x}=f(x)+B u, x\left(t_{0}\right)=x_{0},|u| \leq M, M>0,
$$

The column vector B only has one non-zero cell, i.e. $b_{k}=1, k \in\{1,2, \cdots, n\}$, and $\mathrm{M}$ is constant, which clamps the amplitude of the control action. The constrained control aims to find the control action to meet the constraint of Equ. (4) to drive the chaos system of (3) from the arbitrary initial state $x\left(t_{0}\right)$ to the desired nonstable equilibrium $x(T)=x_{e}$. And $\mathrm{T}$ is the free terminal time. The further requirement is that when the chaos system is driven to the desired non-stable equilibrium, system state was required to stick to that states forever.

Assume the Hamiltonian function of system (4) as

$$
H(x, u, \lambda, t)=\lambda^{T}[f(x)+B u]
$$

where $\lambda \in R^{n}$ is the unknown adjoint variable, which can be defined by the following equation

$$
\dot{\lambda}=-\frac{\partial H}{\partial x}=-\lambda^{T} \frac{\partial f}{\partial x}
$$

In order to identify the optimal control action $\mathrm{u}$, it is necessary for us to come to the helps MPC, then

$$
H\left(x^{*}, u^{*}, \lambda^{*}, t\right) \leq H\left(x^{*}, u^{*}+\delta u, \lambda^{*}, t\right)
$$

And $\delta$ is deployed to represent variation, and the superscript “ $*$ " is assigned to represent the optimal value. To Hamiltonian function (5), under constraint of (7), it is easy for us to get

$$
\left(\lambda^{*}\right)^{T} B u^{*} \leq\left(\lambda^{*}\right)^{T} B u
$$

And the optimal control action comes naturally

$$
u^{*}=-M \operatorname{sgn}\left[\left(\lambda^{*}\right)^{T} B\right]
$$

where sgn demonstrates the sign function mathematically. And in this case, the optimal control is bang-bang format, and the corresponding switching curve is defined as

$$
\left(\lambda^{*}\right)^{T} B=0
$$

To time invariant system (4), Hamiltonian function (5) will keep constant in locus of (10). And we only need to consider the free terminal time constant, then

$$
0=H(x, u, \lambda, t)=\lambda^{T}[f(x)+B u]
$$

Control here aims at stabilizing $x_{e}^{(2)}$ or $x_{e}^{(3)}$ via added outside action $u$ for Equation (4). Here, $\mathrm{M}$ is assigned as 35 , and $x_{0}=[0.1,0,0]^{T}$, in case of completeness, control action added in the first sub-equation $f_{1}$, through the second sub-equation $f_{2}$ and across the third sub-equation $f_{3}$ are covered.

\section{Control actions}

\subsection{Control action added in the first sub-equation}

First of all, the control action added in the first subequation is touched upon. And in this case $B=[1,0,0]^{T}$, from Equ. (10), we have $\lambda_{1}=0, \dot{\lambda}_{1}=0$. If we assume $\left[\lambda_{2}, \lambda_{3}\right]^{T}$ has non-zero solution, then from (6), we can have

$\lambda_{2} \frac{\partial f_{2}}{\partial x_{1}}+\lambda_{3} \frac{\partial f_{3}}{\partial x_{1}}=0$

Connected with (11) and (4), we can have

$$
\lambda_{2} f_{2}+\lambda_{3} f_{3}=0
$$

It is easy to find that the following stands if (12) and (13) are joined verified. 
$S(x)=f_{2} \frac{\partial f_{3}}{\partial x_{1}}-f_{3} \frac{\partial f_{2}}{\partial x_{1}}=c x_{2}^{2}-b x_{3}^{2}+(c-a) b x_{3}=0$

Obviously, $x_{e}^{(2)}$ and $x_{e}^{(3)}$ meets the constraints of Equ. (14), so the two feasible control law can be listed as $\quad u_{+}=-M \operatorname{sgn}[S(x)], u_{-}=M \operatorname{sgn}[S(x)] ; \quad$ where $u_{+}, u_{-}$are assigned to $x_{e}^{(3)}, x_{e}^{(2)}$, separately. The result of $u_{-}$is illustrated in Figure 1.

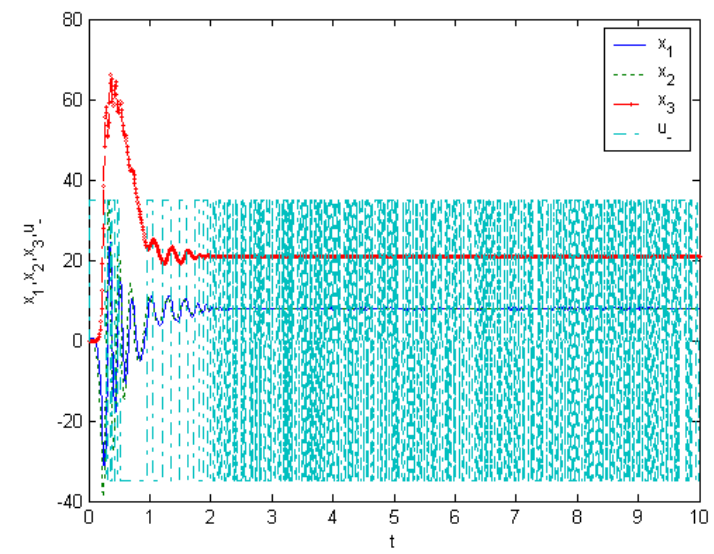

Fig.1. System states and control action transient curves with uadded in the first equation.

And for initial state $x_{0}$, after duration of the transient process, state will be driven to and stabilized on $x_{e}^{(2)}$, and control action switches between -35 and +35 , and even further, because the satisfaction of $x_{e}^{(2)}$ with the switching curve, the switching frequency will become much more often when state comes to the $x_{e}^{(2)}$ nearby. Similarly, the result of $u_{+}$can also enable itself in its stabilizing role, however, in case of space limit, the transient curves with $u_{+}$are omitted here.

\subsection{Control action added in the second sub- equation}

And in case of compactness, only the switching curve is provided here.

$S(x)=f_{1} \frac{\partial f_{3}}{\partial x_{2}}-f_{3} \frac{\partial f_{1}}{\partial x_{2}}=-a x_{1}^{2}+a b x_{3}$

\subsection{Control action added in the third sub- equation}

And in this case, the switching curve is transformed into

$$
S(x)=f_{1} \frac{\partial f_{2}}{\partial x_{3}}-f_{2} \frac{\partial f_{1}}{\partial x_{3}}=a x_{1} x_{2}-a x_{1}^{2}
$$

To be curious, $u_{-}$can not stabilize $x_{e}^{(2)}$, however, $u_{+}$can still hold its capability in stabilizing $x_{e}^{(3)}$, the results about $u_{-}$is illustrated in figure (2), and the result of $u_{+}$is also omitted here.

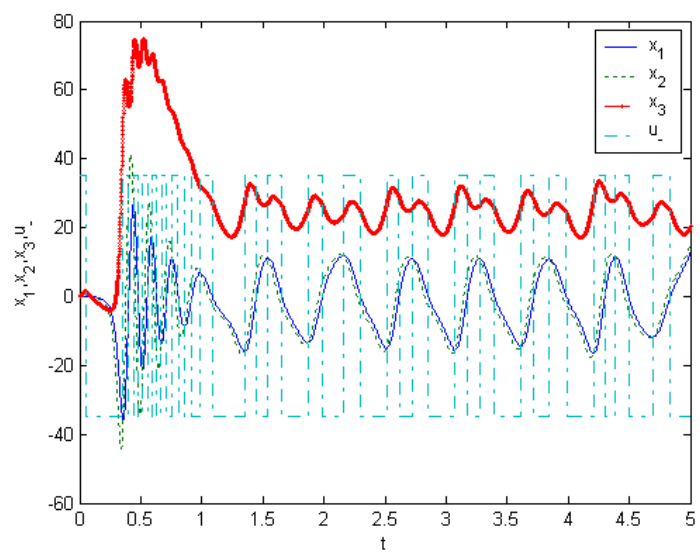

Fig.2. System states and control action transient curves with uadded in the third equation

In order to stabilize $x_{e}^{(2)}$, inspired by the initial sensitive peculiarity of chaos system, the sub-control action $u_{-}$is segmented further into

$$
u_{-}=\left\{\begin{array}{l}
u_{+} ;\left|x_{1}-\mathrm{c}\right| \leq \sigma \\
0 ;\left|x_{1}-\mathrm{c}\right|>\sigma .
\end{array}\right.
$$

where $x_{1}$ is the first sub-state variable of chaos system, while $\mathrm{c}$ and $\sigma$ are constants, and $\mathrm{c}$ and be chosen as $x_{\mathrm{e} 1}^{(2)}$ itself, $\sigma$ can be viewed as the threshold value, here we assumed as 2, and after this kind of reform, the result of $u_{-}$is illustrated as figure (3).
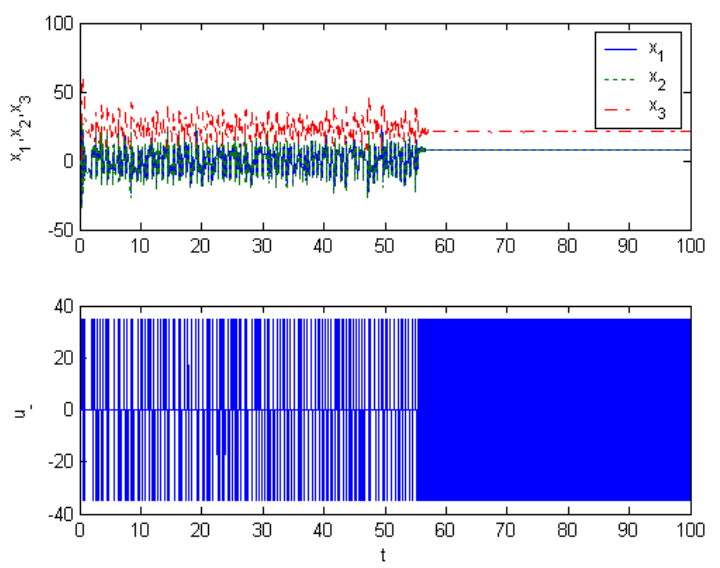

Fig.3. System states and control action transient curves with improved $\mathrm{u}$ - added in the third equation 
And by this wait to go style, the sub-equation based control can fully functions for all the non-stable equilibrium.

\section{Conclusion and some prospects}

In this paper, inspired by the initial value sensitive peculiarity of the chaos system, in helps of the MPC, the wait to go style sub-control action is introduced extra to make it possible to realize the full scale stabilization of the non-stable equilibrium of Chen chaos system.

Chaos system is not alone in complex behavior, and it is not only the one to be toughly manipulated with, high performance vehicle control pioneered in Reference (4) and the delicate manner of regulation in Reference (5), can be verifications of this. As the large scale complex systems, not the plant itself, but also the harsh environment surrounded, such as time varying delay, incomplete, noisy and unreliable information, annoy disturbances, and multi-inputs multi-outputs, just mention a few, set the obstacles to be overcome, under these unfriendly conditions, adaptive schemes always be the clever choice, please referring to Reference (6). For the individuals large quantity system, like multi-agent systems, control philosophy has changed sharply, and the main concentration has been shifted from hardware to software, and contemporarily from both of them to the wetware, and that pursues eagerly in learning from the social behavior of group habited living beings and human society system, and without hesitation, this can be said as a big leap in control in complex systems arrangement, please see in Reference (7-9). So, the orchestrated strategy demonstrated here deserves its further exploration in the near future, to the plant we approach, the task we try to manipulate with, the adversary disturbance we must face, all of these must be balanced via trade-off negotiation, co-operation, and regulation.

\section{Acknowledgements}

This work is partially supported by NSFC Grant (61340041 and 61374079) and the Project-sponsored by SRF for ROCS, SEM to Yunzhong Song.

\section{References}

1. Yuchu Tian, M. Tade and D.Levy, Constrained control of chaos, Physics Letters A, 296 (2002) 87-90.

2. Yunzhong Song, Guangzhou Zhao and Donglian Qi, Some comments on constrained control of chaos, Physics Letters A, 359 (2006) 624-626.

3. Guanrong Chen and T.Ueta, Yet another chaotic attractor, International Journal of Bifurcation and Chaos, 9 (1999) 1465-1466.

4. Yingmin Jia, Robust control with decoupling performance for steering and traction of $4 \mathrm{WS}$ vehicles under velocity-varying motion, IEEE Transactions on Control Systems Technology, 8(2000) 554-569.

5. Yingmin Jia, Alternative proofs for improved LMI representations for the analysis and the design of continuous-time systems with polytopic type uncertainty: a predictive approach, IEEE Transactions on Automatic Control, 48(2003) 1413-1416.

6. Yingmin Jia, General solution to diagonal model matching control of multi-output-delay systems and its applications in adaptive scheme, Progress in Natural Science, 19( 2009), 79-90.

7. Yunzhong Song, Ziyi Fu, Fuzhong Wang, Socialized multi-agent system rendezvous via networks of networks, Journal of Robotics, Networking and Artificial Life, 3 (2016) 136-139.

8. Yunzhong Song, Consensus of agents with mixed linear discrete dynamics, International Journal of Control, Automation and Systems, 14 (2016) 1139-1143.

9. Deyuan Meng, Yingmin Jia, Robust consensus algorithms for multiscale coordination control of multivehicle systems with disturbances, IEEE Transactions on Industrial Electronics, 63 (2016) 1107-1119. 